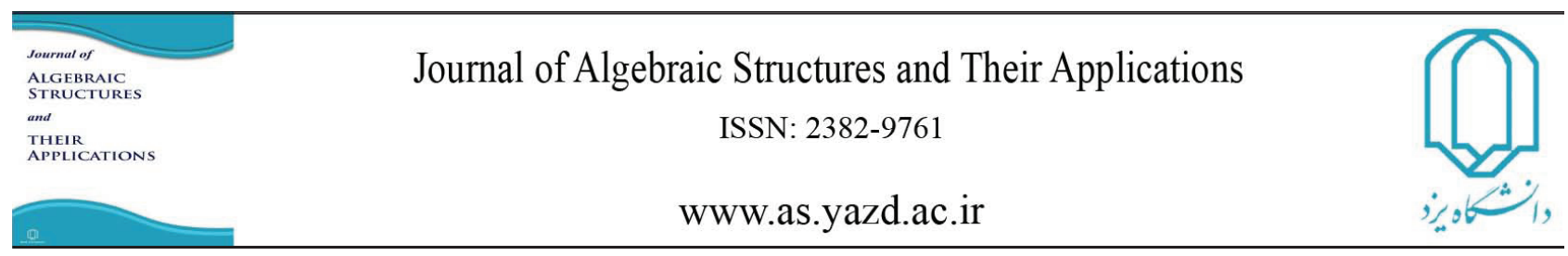

Algebraic Structures and Their Applications Vol. 4 No. 2 ( 2017 ) pp 15-25.

\title{
ON THE ZERO FORCING NUMBER OF SOME CAYLEY GRAPHS
}

\author{
E. VATANDOOST* AND Y. GOLKHANDY POUR
}

Communicated by A.R. Ashrafi

\begin{abstract}
Let $\Gamma$ be a graph whose each vertex is colored either white or black. If $u$ is a black vertex of $\Gamma$ such that exactly one neighbor $v$ of $u$ is white, then $u$ changes the color of $v$ to black. A zero forcing set for a graph $\Gamma$ is a subset of vertices $Z \subseteq V(\Gamma)$ such that if initially the vertices in $Z$ are colored black and the remaining vertices are colored white, then $Z$ changes the color of all vertices in $\Gamma$ to black. The zero forcing number of $\Gamma$ is the minimum of $|Z|$ over all zero forcing sets for $\Gamma$ and is denoted by $Z(\Gamma)$.

In this paper, we consider the zero forcing number of some families of Cayley graphs. In this regard, we show that $Z\left(\operatorname{Cay}\left(D_{2 n}, S\right)\right)=2|S|-2$, where $D_{2 n}$ is dihedral group of order $2 n$ and $S=\left\{a, a^{3}, \ldots, a^{2 k-1}, b\right\}$. Also, we obtain $Z(C a y(G, S))$, where $G=\langle a\rangle$ is a cyclic group of even order $n$ and $S=\left\{a^{i}: 1 \leq i \leq n\right.$ and $i$ is odd $\}, S=\left\{a^{i}: 1 \leq i \leq\right.$ $n$ and $i$ is odd $\} \backslash\left\{a^{k}, a^{-k}\right\}$ or $|S|=3$.
\end{abstract}

DOI:http://dx.doi.org/10.29252/asta.4.2.15

MSC(2010): Primary: 05C25. Secondary: 05C75.

Keywords: Zero forcing number, Cayley graph, Algorithm.

Received: 9 December 2017, Accepted: 16 May 2018

*Corresponding author

(C) 2017 Yazd University. 


\section{INTRODUCTION}

In this paper all graphs are assumed to be finite, simple and undirected. We will often use the notation $\Gamma=(V, E)$ to denote the graph with non-empty vertex set $V=V(\Gamma)$ and edge set $E=E(\Gamma)$. An edge of $\Gamma$ with endpoints $u$ and $v$ is denoted by $u v$, and we say $u \sim v$ if $u v \in E(\Gamma)$ and $u \nsim v$, otherwise. Vertex $v$ is neighbor of vertex $u$ if $u v \in E(\Gamma)$, and the set of all neighbors of $u$ is denoted by $N(u)$. The degree of a vertex $u$ is $|N(u)|$ and is denoted by $\operatorname{deg}(u)$. The minimum degree over all the vertices of a graph $\Gamma$ is the minimum degree of graph $\Gamma$ and is denoted by $\delta(\Gamma)$. If $S \subset V(\Gamma)$, then induced subgraph on $S$ in the graph $\Gamma$ is denoted by $\Gamma[S]$.

Graph parameters have many application in other sciences trends. As one of the most important, it can be zero forcing number. Also, zero forcing variants have applications in network theory, logic and quantum, see [II]],[I4] and [9] for more details in these topics. If $\Gamma$ is a graph with each vertex colored either white or black, $u$ is a black vertex of $\Gamma$, and exactly one neighbor $v$ of $u$ is white, then $u$ change the color of $v$ to black. This algorithm is called the color-change rule in the graph $\Gamma$. Given a coloring of $\Gamma$, the derived coloring is the (unique) result of applying the color-change rule until no more changes are possible. A zero forcing set for a graph $\Gamma$ is a subset of vertices $Z \subseteq V(\Gamma)$ such that if initially the vertices in $Z$ are colored black and the remaining vertices are colored white, then the derived coloring of $\Gamma$ is all black. $Z(\Gamma)$ is the minimum of $|Z|$ over all zero forcing sets for $\Gamma$.

The computation of zero forcing number is very difficult and is NP-hard, see [ 8$]$. The zero forcing number was first introduced by F. Barioli et al. (AIM Minimum Rank Work Group) [2] in 2008, and they used this parameter for providing an upper bound for the maximum nullity of a graph. In [7] Davila and Kenter conjectured the lower bound

$$
Z(\Gamma) \geq(g-2)(\delta(\Gamma)-2)+2
$$

for every graph $\Gamma$ of girth $g$ at least 3 and minimum degree $\delta(\Gamma)$ at least 2 . This conjecture was considered by Gentner [II]], for $g=4$ and for triangle free graphs. Also, Gentner and Rautenbach in [U] proved this conjecture for $g \in\{5,6\}$. In [ [ $]$, Davila and Kenter shown that $Z(\Gamma) \geq 2 \delta(\Gamma)-2$ for graphs with girth of at least 5 .

Let $G$ be a group and $S$ be a subset of $G$ which is closed under taking inverse and does not contain the identity element $e$. The Cayley $\operatorname{graph} \operatorname{Cay}(G, S)$ is a graph with vertex set $G$ and edge set $\left\{u v: v u^{-1} \in S\right\}$. Cayley graphs are regular and vertex transitive.

In this paper, we consider the zero forcing number of some families of Cayley graphs $\operatorname{Cay}(G, S)$. We show that $Z\left(\operatorname{Cay}\left(D_{2 n}, S\right)\right)=2|S|-2$, where $D_{2 n}$ is dihedral group of order $2 n$ and $S=\left\{a, a^{3}, \ldots, a^{2 k-1}, b\right\}$. Also, we prove that if $G=\langle a\rangle$ is a cyclic group of even order $n$, then $Z(\operatorname{Cay}(G, S))=2|S|-2$, where $S=\left\{a^{i}: i\right.$ is odd $\}, S=\left\{a^{i}: i\right.$ is odd $\} \backslash\left\{a^{k}, a^{-k}\right\}$ 
or $S=\left\{a^{i}, a^{-i}, a^{n / 2}\right\}$. In this regard, it seems that if induced subgraph on $S$ is empty, then $Z(\operatorname{Cay}(G, S)) \geq 2|S|-2$.

\section{PRELIMINARIES}

In this section, some algebraic properties of Cayley graphs are studied which are used to prove our main results.

Lemma 2.1. Let $G$ be a finite group and $S$ be a subset of $G$ such that $S=S^{-1}$ and $e \notin S$. If $H$ is a subgroup of $G$, then induced subgraph on all cosets of $H$ in $C a y(G, S)$ are isomorphic.

Proof. Let $H g$ be a coset of $H$, where $g \in G$. For some $u, v \in H g$, there exist $h_{1}, h_{2} \in H$ such that $u=h_{1} g$ and $v=h_{2} g$. Hence $u v^{-1}=h_{1} h_{2}^{-1}$. Thus two vertices $u$ and $v$ are adjacent if and only if $h_{1}$ and $h_{2}$ are adjacent.

Abdollahi and Vatandoost [I] proved the following lemma.

Lemma 2.2. [T] Let $G$ be a group and $G=\langle S\rangle$, where $S=S^{-1}$ and $e \notin S$. If $a \in S$ and $O(a)=m>2$, then $\operatorname{Cay}(G, S)$ has the cycle with $m$ vertices as a subgraph.

Lemma 2.3. Let $G$ be a group, $S$ be a subset of $G$ such that $S=S^{-1}$ and $e \notin S$. For each $u \in \operatorname{Cay}(G, S)[S]$, if $\operatorname{deg}_{\operatorname{Cay}(G, S)[S]}(u) \leq|S|-3$, then $Z(\operatorname{Cay}(G, S))>|S|$.

Proof. Since $\operatorname{Cay}(G, S)$ is $|S|$-regular, $Z(\operatorname{Cay}(G, S)) \geq|S|$. On the contrary, let $Z(\operatorname{Cay}(G, S))=|S|$. Then we have a set of size $|S|$ as the set of initial black vertices. Since $\operatorname{Cay}(G, S)$ is a vertex-transitive graph, without loss of generality assume that $e$ is the first black vertex which is performing a force in a zero forcing process. Hence all members of $S$ are in $Z$ except one of them which is forced by $e$. Since for each $u \in \operatorname{Cay}(G, S)[S]$, $\operatorname{deg}_{C a y(G, S)[S]}(u) \leq|S|-3$, each vertex in $S$ is adjacent with at least other two white neighbors. Thus, there is no vertex whose perform a force in a zero forcing process, which is a contradiction. Therefore, the initial set of black vertices can not be a zero forcing set for $\operatorname{Cay}(G, S)$.

Example 2.4. Let $G=\langle a\rangle$ whose $O(a)=6$ and $S=\left\{a, a^{3}, a^{5}\right\}$. Then $\operatorname{Cay}(G, S)$ is isomorphic to the graph $\Gamma$ which is drawn in Figure $\mathbb{0}$.

In this case, all conditions are as Lemma [2.3, and so $Z(\operatorname{Cay}(G, S))>3$. On the other hand, it is easy to check that $Z=\left\{e, a, a^{2}, a^{3}\right\}$ is a zero forcing set for $\operatorname{Cay}(G, S)$ and so $Z(\operatorname{Cay}(G, S)) \leq 4$. Thus, $Z(\operatorname{Cay}(G, S))=4$.

For the main results of this paper, we need the following useful theorem. 


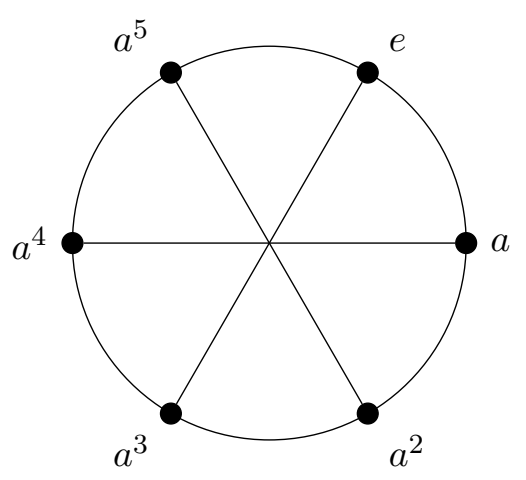

FIGURE 1. The graph $C a y(\langle a\rangle, S)$, where $O(a)=6$ and $S=\left\{a, a^{3}, a^{5}\right\}$

Theorem 2.5. [2] Let $K_{n_{1}, n_{2}, \ldots, n_{k}}$ be a complete multipartite graph with at least one $n_{i}>1$ for $1 \leq i \leq k$. Then $Z\left(K_{n_{1}, n_{2}, \ldots, n_{k}}\right)=\left(n_{1}+n_{2}+\ldots+n_{k}\right)-2$.

\section{MAin Results}

At first, we provide the following lemma on Cayley graphs which will be used in the sequel.

Theorem 3.1. Let $G$ be a group of even order $n$ and $S \subseteq G$ such that $S=S^{-1}, e \notin S$ and $|S|=n / 2$.

i. $\operatorname{Cay}(G, S)[S]=\emptyset$ if and only if $G \backslash S$ is a subgroup of $G$.

ii. If $\operatorname{Cay}(G, S)[S]=\emptyset$, then $Z(\operatorname{Cay}(G, S))=n-2$.

Proof. (i) If $G \backslash S$ is a subgroup of $G$, then for $u, v \in G \backslash S$, we have $u v^{-1} \in G \backslash S$ and so $u$ is not adjacent to $v$. Since $\operatorname{Cay}(G, S)$ is $n / 2$-regular, any vertex contained in $G \backslash S$ is adjacent to all vertices contained in $S$ and vice versa. Hence induced subgraph on $S$ must be empty. Conversely, let $\operatorname{Cay}(G, S)[S]=\emptyset$. Then each vertex contained in $S$ is adjacent to all vertices contained in $G \backslash S$. So induced subgraph on $G \backslash S$ is empty. Therefore for each $u, v \in G \backslash S$, $u v^{-1} \in G \backslash S$ and so $G \backslash S$ is a subgroup of $G$.

(ii) By (i), If $\operatorname{Cay}(G, S)[S]=\emptyset$, then $G \backslash S$ is a subgroup of $G$. Thus $\operatorname{Cay}(G, S)$ is isomorphic to the complete bipartite graph with two bipartition sets of size $n / 2$. Hence Theorem 2.5 implies that $Z(\operatorname{Cay}(G, S))=n-2=2|S|-2$.

Corollary 3.2. Let $G=\langle S\rangle$ be a cyclic group of even order $n$ and $S=\left\{a^{i}: i\right.$ is an odd $\}$. Then $Z(\operatorname{Cay}(G, S))=2|S|-2$.

Theorem 3.3. Let $G=\langle a\rangle$ be a cyclic group of order $n$ and $S=\left\{a^{i}: i\right.$ is an odd $\} \backslash\left\{a^{k}, a^{-k}\right\}$, where $o\left(a^{2 k}\right)>2$. Then $Z(\operatorname{Cay}(G, S))=2|S|-2$. 
Proof. Define $A=\left\{a^{i}: i\right.$ is an odd $\}$ and $B=\left\{a^{i}: i\right.$ is an even $\}$. Since $o\left(a^{k}\right) \neq 2$, we have $a^{k+1} \neq a^{1-k}$. Also, $N\left(a^{k+1}\right)=A \backslash\left\{a, a^{1+2 k}\right\}$ and $N\left(a^{1-k}\right)=A \backslash\left\{a, a^{1-2 k}\right\}$. Since $o\left(a^{k}\right) \neq 4$, we have $a^{1+2 k} \neq a^{1-2 k}$. Suppose that

$$
Z=(S \backslash\{a\}) \cup\left(B \backslash\left\{a^{2}, a^{1+k}, a^{1-k}\right\}\right)
$$

is a set of black vertices in $\operatorname{Cay}(G, S)$ and remaining vertices are white. After applying the zero forcing process, $a$ is forced by $e, a^{1+k}$ is forced by $a^{1-2 k}, a^{1-k}$ is forced by $a^{1+2 k}$. On the other hand, $o\left(a^{k}\right) \neq 4$ implies that $a^{2 k}$ forces $a^{-k}$ and $a^{-2 k}$ forces $a^{k}$. Thus, $Z$ is a zero forcing set for $\operatorname{Cay}(G, S)$ and so $Z(\operatorname{Cay}(G, S)) \leq n-6$.

On the other hand, let $Z$ be a zero forcing set for $\operatorname{Cay}(G, S)$ with minimum cardinality. Since $\operatorname{Cay}(G, S)$ is a vertex transitive graph, with no loss of generality assume that $e$ is the first forcing vertex whose perform a force in a zero forcing process. Thus, there exist $C \subseteq Z \cap S$ such that $|C|=|S|-1$. Without loss of generality, let $a \notin C$. By applying the forcing process, $a$ is forced by $e$. In continue, let $u$ be the second vertex whose perform a force in a zero forcing process. The following cases will be considered.

Case 1. $u=a^{k}$ or $u=a^{-k}$.

In this case, it is essential that $B \backslash\left\{a^{2 k}, a^{i}\right\} \subseteq Z$ or $B \backslash\left\{a^{-2 k}, a^{j}\right\} \subseteq Z$, for $i \neq 2 k$ and $j \neq-2 k$, respectively. Hence, $|Z| \geq n-4$, which contradicts the fact that $|Z| \leq n-6$.

Case 2. $u \in B \backslash\left\{a^{2 k}, a^{-2 k}\right\}$.

With no loss of generality, assume that $u=a^{2}$. Thus, we will necessarily have $a^{k} \in Z$. By applying the forcing process, $a^{-k}$ is forced by $a^{2}$. Here, we have no black vertex with only one white neighbour and so it is necessary to change the color of another vertex. Let $v$ be the third forcing vertex whose perform a force in a zero forcing process. If $v \in B$, then $v$ perform no force. The following subcases will be considered.

Subcase 2.1. $v=a^{k}$ or $v=a^{-k}$.

In this case, it is essential that $B \backslash\left\{a^{2 k}, a^{i}\right\} \subseteq Z$ or $B \backslash\left\{a^{-2 k}, a^{j}\right\} \subseteq Z$, for $i \neq 2 k, 2$ and $j \neq-2 k, 2$, respectively. Hence, $|Z| \geq n-4$, which contradicts the fact that $|Z| \leq n-6$.

Subcase 2.2. $v \in A \backslash\left\{a^{k}, a^{-k}\right\}$.

Without loss of generality, assume that $v=a$. Thus, we will necessarily have $B \backslash$ $\left\{a^{3}, a^{1+k}, a^{1-k}\right\} \subseteq Z$. Hence, $|Z| \geq n-5$, which contradicts the fact that $|Z| \leq n-6$.

Case 3. $u=a^{2 k}$.

After applying the forcing process, $a^{-k}$ is forced by $a^{2 k}$. Since no black vertex has only one white neighbour, it is necessary to change the color of another vertex to black. Assume that $v$ is the third forcing vertex whose perform a force in a zero forcing process. The following subcases will be considered.

Subcase 3.1. $v \in B \backslash\left\{a^{-2 k}\right\}$ or $v=a^{-2 k}$. 
First suppose that $v \in B \backslash\left\{a^{-2 k}\right\}$. After applying the forcing process, $a^{k}$ is forced by $v$ and $a^{-2 k}$ is forced by $a^{k}$. Next assume that $v=a^{-2 k}$. Thus, after applying the forcing process, $a^{k}$ is forced by $a^{-2 k}$. Hence, the conditions are similar for both cases. If $a^{k}$ or $a^{-k}$ is the fourth forcing vertex, then it is necessary to have $B \backslash\left\{a^{j}\right\} \subseteq Z$, where $a^{j} \notin\left\{e, v, a^{2 k}, a^{-2 k}\right\}$. Hence, $|Z| \geq n-4$, which contradicts the fact that $|Z| \leq n-6$. Also, if the fourth forcing vertex is contained in $B \backslash\left\{a^{2 k}, a^{-2 k}\right\}$, then it can perform no force.

In addition, assume that the fourth forcing vertex is located in $A \backslash\left\{a^{k}, a^{-k}\right\}$. Without loss of generality, let $a$ be the fourth forcing vertex which is not located in $\left\{a^{3 k}, a^{-3 k}\right\}$. Then we will necessarily have $B \backslash\left\{a^{2}, a^{1+k}, a^{1-k}\right\} \subseteq Z$. On the other hand, either $a^{1+k} \in Z$ or $a^{1-k} \in Z$, then $a^{-2 k}$ can not be forced by $a^{k}$, which is a contradiction. Hence $B \backslash\left\{a^{2}\right\} \subseteq Z$ and so $|Z| \geq n-5$, which contradicts the fact that $|Z| \leq n-6$. Now, let $a^{3 k} \in A \backslash\left\{a^{k}, a^{-k}\right\}$ be the fourth forcing vertex whose perform a force. If $o\left(a^{3 k}\right)=2$, then $a^{2 k}, a^{-2 k} \notin N\left(a^{3 k}\right)$ and so essentially $B \backslash\left\{a^{j}\right\} \subseteq Z$, where $a^{j} \notin\left\{e, a^{2 k}, a^{-2 k}\right\}$. Hence $|Z| \geq n-4$, which contradicts the fact that $|Z| \leq n-6$. Also, if $o\left(a^{3 k}\right) \neq 2$, then $a^{2 k} \notin N\left(a^{3 k}\right)$ and $a^{-2 k} \in N\left(a^{3 k}\right)$. Thus, we will necessarily have $B \backslash\left\{a^{j}\right\} \subseteq Z$, where $a^{j} \notin\left\{e, a^{2 k}, a^{-2 k}\right\}$. Hence $|Z| \geq n-4$, which contradicts the fact that $|Z| \leq n-6$.

Subcase 3.2. $v=a^{k}$.

In this case, it is essential that $B \backslash\left\{a^{2 k}, a^{i}\right\} \subseteq Z$, for $i \neq 2 k$. Hence, $|Z| \geq n-3$, which contradicts the fact that $|Z| \leq n-6$.

Subcase 3.3. $v \in A \backslash\left\{a^{k}, a^{-k}\right\}$.

Without loss of generality, assume that $v=a$. Thus, we will necessarily have $B \backslash$ $\left\{a^{2}, a^{1+k}, a^{1-k}\right\} \subseteq Z$. Hence, $|Z| \geq n-6$. On the other hand, by performing a force, $a^{2}$ is forced by $a, a^{1+k}$ is forced by $a^{1-2 k}, a^{1-k}$ is forced by $a^{1+2 k}$. Also, $a^{k}$ is forced by $a^{2}$. Thus, $Z=\left(A \backslash\left\{a, a^{k}, a^{-k}\right\}\right) \cup\left(B \backslash\left\{a^{2}, a^{1+k}, a^{1-k}\right\}\right)$ is a zero forcing set for $\operatorname{Cay}(G, S)$ and so $|Z| \leq n-6$. Therefore $Z(C a y(G, S))=n-6=2|S|-2$.

Case 4. $u \in A \backslash\left\{a^{k}, a^{-k}\right\}$.

Without loss of generality, assume that $u=a$. Thus, we will necessarily have $B \backslash$ $\left\{a^{2}, a^{1+k}, a^{1-k}\right\} \subseteq Z$ and so $|Z| \geq n-6$. On the other hand, by performing a force, $a^{2}$ is forced by $a, a^{1+k}$ is forced by $a^{1-2 k}, a^{1-k}$ is forced by $a^{1+2 k}$. On the other hand, $o\left(a^{k}\right) \neq 4$ implies that $a^{2 k}$ forces $a^{-k}$ and $a^{-2 k}$ forces $a^{k}$. Thus, $Z=\left(A \backslash\left\{a, a^{k}, a^{-k}\right\}\right) \cup\left(B \backslash\left\{a^{2}, a^{1+k}, a^{1-k}\right\}\right)$ is a zero forcing set for $\operatorname{Cay}(G, S)$ and so $|Z| \leq n-6$. Therefore $Z(\operatorname{Cay}(G, S))=n-6=2|S|-2$.

Theorem 3.4. Let $G=\langle a\rangle$ be a cyclic group of order $n=4 k$. If $k>1$ is odd and $S=\left\{a^{i}\right.$ : $i$ is odd and $1 \leq i \leq 4 k-1\}$, then $Z(\operatorname{Cay}(G, S))=n-4$. 
Proof. Define $V_{1}=\{e, 2 k\}, V_{2}=S, V_{3}=\left\{a^{i}: i\right.$ is even and $\left.2 \leq i \leq 4 k-2\right\} \backslash\left\{a^{2 k}\right\}$ and $V_{4}=\left\{a^{k}, a^{-k}\right\}$. It is easy to check that $N(e)=N\left(a^{2 k}\right)=S, N\left(a^{k}\right)=N\left(a^{-k}\right)=V_{3}$, $N\left(a^{i}\right)=V_{3} \backslash\left\{a^{i+k}, a^{i-k}\right\} \cup V_{1}$ and $N\left(a^{j}\right)=V_{2} \backslash\left\{a^{j+k}, a^{j-k}\right\} \cup V_{4}$, where $i$ is odd and $j$ is

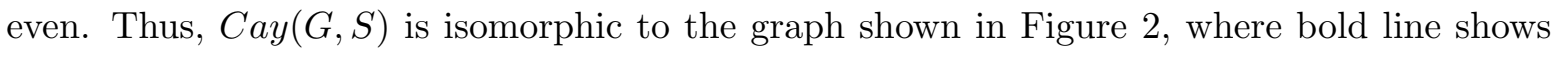
that each vertex is adjacent to all other vertices contained in the opposite set and dashed line shows that each vertex is not adjacent to exactly two vertices contained in the opposite set.

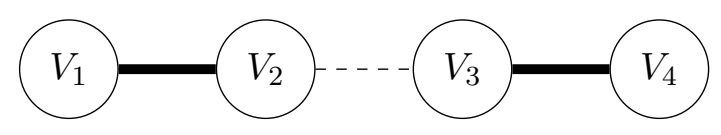

Figure 2. The graph $\operatorname{Cay}(G, S)$, where $G=\langle a\rangle$ and $S=\left\{a^{i}: i\right.$ is odd and $\left.1 \leq i \leq 4 k-1\right\}$.

Let $Z=V(\operatorname{Cay}(G, S)) \backslash\left\{a, a^{4 k-2}, a^{k+1}, a^{-k}\right\}$. By applying the zero forcing process, $a$ is forced by $e, a^{4 k-2}$ is forced by $a, a^{-k}$ is forced by $a^{2}$ and $a^{k+1}$ is forced by $a^{k}$. Thus, $Z$ is a zero forcing set for $\operatorname{Cay}(G, S)$ and so $Z(\operatorname{Cay}(G, S)) \leq 4 k-4=n-4$.

Now, let $Z(\operatorname{Cay}(G, S)) \leq 4 k-5$ and $Z$ be a zero forcing set with minimum cardinality for $\operatorname{Cay}(G, S)$. Since $\operatorname{Cay}(G, S)$ is vertex transitive graph, we can assume that $e \in Z$. Then it is essential that the color of all vertices in $V_{2}$ except one vertex, say $a$ changed to black. Hence, $a$ is forced by $e$. Here, we have at least other four white vertices which are located in $V_{1}, V_{3}$ and $V_{4}$. If four white vertices are contained in $V_{3}$, then there is no black vertices with exactly one white neighbour whose perform a force in a zero forcing process. Since every vertices in $V_{3}$ is adjacent to $V_{4}$, it is necessary that $a^{k} \in Z$ or $a^{-k} \in Z$. Without loss of generality, assume that $a^{k} \in Z$. The following cases will be considered.

Case 1. There exist three white vertices in $V_{3}$. Then $a^{-k} \notin Z$. Furthermore, white vertices located in $V_{3}$ are $a^{i+k}, a^{i-k}$ and $a^{j}$, where $i$ is odd and $j \neq 2 k$ is even. Hence, $a^{j}$ is forced by $a^{i}$. It is clear that if $1 \leq j \leq 4 k-1$ is odd and $a^{i+k} \in N\left(a^{j}\right)$, then $a^{i-k} \in N\left(a^{j}\right)$. So, any vertices in $V_{2}$ and $V_{4}$ can not forces the vertices $a^{i+k}$ and $a^{i-k}$, which is wrong.

Case 2. Let $a^{2 k}$ and $a^{-2 k}$ be white vertices. then there exist at least other two white vertices say $x$ and $y$. Hence, there exist an odd $1 \leq i \leq 4 k-1$ such that $x=a^{i+k}$ and $y=a^{i-k}$. Since $a^{2 k}$ is the only white neighbour of $a^{i}, a^{2 k}$ is forced by $a^{i}$. It is clear that if $1 \leq j \leq 4 k-1$ is odd and $a^{i+k} \in N\left(a^{j}\right)$, then $a^{i-k} \in N\left(a^{j}\right)$. So, there is no black vertex whose perform a force in a zero forcing process. Therefore, $Z(\operatorname{Cay}(G, S)) \geq n-4$ and so $Z(\operatorname{Cay}(G, S))=n-4$.

Theorem 3.5. Let $D_{2 n}=\left\langle a, b: a^{n}=b^{2}=(a b)^{2}=e\right\rangle$ be the dihedral group of order $2 n$, where $n=2 k$. Also, let $S=\left\{a, a^{3}, \ldots, a^{2 k-1}, b\right\}$. Then $Z\left(\operatorname{Cay}\left(D_{2 n}, S\right)\right)=2|S|-2$. 
Proof. Define $A_{1}=\left\{a^{i}: 1 \leq i \leq n, i\right.$ is an even $\}, A_{2}=\left\{a^{j}: 1 \leq j \leq n, j\right.$ is an odd $\}$, $A_{3}=\left\{b a^{i}: 1 \leq i \leq n, i\right.$ is an even $\}$ and $A_{4}=\left\{b a^{j}: 1 \leq j \leq n, j\right.$ is an odd $\}$. It is easy to see that $\operatorname{Cay}\left(D_{2 n}, S\right)$ is isomorphic to the graph shown in Figure B, where bold line shows that each vertex is adjacent to all other vertices contained in the opposite set and dashed line shows that each vertex is adjacent to exactly one vertex contained in the opposite set. Also, induced subgraph on $A_{i}$ is empty, for $1 \leq i \leq 4$. Since induced subgraph on $S$ is empty, Lemma $[2.3]$ implies that $Z\left(\operatorname{Cay}\left(D_{2 n}, S\right)\right)>|S|$.

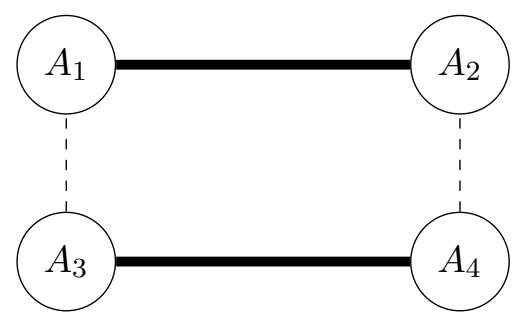

FIGURE 3. The graph $\operatorname{Cay}\left(D_{2 n}, S\right)$, where $S=\left\{a, a^{3}, \ldots, a^{2 k-1}, b\right\}$.

Suppose that $Z$ is the set of minimum size whose performing a force in a zero forcing process. Since Cayley graph is vertex transitive, suppose that $e \in Z$ and with no loss of generality assume that $e$ is the first black vertex which perform a force in a zero forcing process. Essentially, $A_{2} \subseteq Z$ or $\left|A_{2} \backslash Z\right|=1$. Either $A_{2} \subseteq Z$ or $a \in A_{2} \backslash Z$, then after applying the zero forcing process, the vertex $e$ change the color of $b \in A_{3}$ or $a \in A_{2}$ to black, respectively. Thus, with no loss of generality assume that $A_{2} \cup\{e\} \subseteq Z$.

In continue, there is no black vertex with only one white neighbour and so no vertex can apply a forcing process. Let $u$ be the next vertex that start the forcing process. If $u \in A_{2}$, then $A_{1} \backslash\{e\}$ must be colored black. Also, if $u \in A_{3}$, then all vertices in $A_{4}$ except one vertex must be colored black. Next, if $u \in A_{4}$, then $u$ and all vertices in $A_{3} \backslash\{b\}$ except one vertex must be colored black. Finally, if $u \in A_{1}$, then $u$ can change the color of only one vertex contained in $A_{3}$ to black, and so we must choose all vertices in $A_{1}$ until they change the color of all vertices in $A_{3}$. Until now, any choice for $u$ forces us for adding other $k-1$ black vertices to $Z$. Thus, $|Z| \geq 2 k$. On the other hand, it is not hard to check that the set $Z=A_{1} \cup A_{2}$ is a zero forcing set for $\operatorname{Cay}(G, S)$. Hence $Z\left(\operatorname{Cay}\left(D_{2 n}, S\right)\right) \leq 2 k$. Therefore $Z\left(\operatorname{Cay}\left(D_{2 n}, S\right)\right)=2 k=2|S|-2$.

Theorem 3.6. Let $G=\langle g\rangle$ be a finite cyclic group of even order $n$ and let $S$ be a generating subset of $G$ such that $|S|=3$ and $S=\left\{g^{i}, g^{-i}, g^{n / 2}\right\}$. If $G$ is isomorphic to $\mathbb{Z}_{4}$ or $\mathbb{Z}_{6}$ and $i=2$, then $Z(\operatorname{Cay}(G, S))=3$. Otherwise, $Z(\operatorname{Cay}(G, S))=2|S|-2=4$. 
Proof. If $G \cong \mathbb{Z}_{4}$, then $\operatorname{Cay}(G, S)$ is isomorphic to $K_{4}$ and so $Z(\operatorname{Cay}(G, S))=3$. If $G \cong \mathbb{Z}_{6}$ and $i=1$, then by Lemma [2.3, $Z(\operatorname{Cay}(G, S)) \geq 4$. Also, let $Z=\left\{e, a, a^{3}, a^{2}\right\}$ be a set of black vertices. Then $e$ forced $a^{3}$ and $a$ forced $a^{4}$. Thus $Z$ is a zero forcing set for $\operatorname{Cay}(G, S)$ and so $Z(\operatorname{Cay}(G, S)) \leq 4$. Hence $Z(\operatorname{Cay}(G, S))=4$. Also, if $G \cong \mathbb{Z}_{6}$ and $i=2$, then $Z(C a y(G, S)) \geq 3$. On the other hand, if $Z=\left\{e, a^{2}, a^{4}\right\}$ is a set of black vertices, then $e$ forced $a^{3}, a^{2}$ forced $a^{5}$ and $a^{4}$ forced $a$. Hence $Z$ is a zero forcing set for $\operatorname{Cay}(G, S)$ and so $Z(\operatorname{Cay}(G, S)) \leq 3$. Hence $Z(\operatorname{Cay}(G, S))=3$.

Now, let $n>6$. Since $S$ is a generating subset of $G$, we have $\operatorname{gcd}(i, n)=1 \operatorname{or} \operatorname{gcd}(i, n / 2)=1$.

Case 1. $\operatorname{gcd}(i, n)=1$.

In this case, $O\left(g^{i}\right)=n$ and so $G$ is generated by $g^{i}$. By Lemma [2.2, $\operatorname{Cay}(G, S)$ has an $n$ cycle as a subgraph. Define $T_{\ell}=\left\{g^{\ell i}, g^{(\ell+n / 2) i}\right\}$ and Observe that for each $1 \leq s<t \leq n$, $T_{s} \cap T_{t}=\emptyset$. Since $g^{(\ell+n / 2) i} g^{-\ell i}=g^{n / 2 i}=g^{n / 2} \in S$, induced subgraph on $T_{\ell}$ is isomorphic to $P_{2}$. In this case, $\operatorname{Cay}(G, S)$ is isomorphic to a cubic graph with an even number of vertices, formed from an $n$-cycle by adding edges connecting opposite pairs of vertices in the cycle. In this case, by Lemma [2.3], $Z(\operatorname{Cay}(G, S)) \geq 4$. On the other hand, if $Z=\left\{e, g^{i}, g^{-i}, g^{n / 2}\right\}$, then it is easy to check that $Z$ is a zero forcing set for $\operatorname{Cay}(G, S))$ and so $Z(\operatorname{Cay}(G, S)) \leq 4$. Therefore, $Z(\operatorname{Cay}(G, S))=4$.

Case 2. $\operatorname{gcd}(i, n) \neq 1$ but $\operatorname{gcd}(i, n / 2)=1$.

Since $\operatorname{gcd}(i, n / 2)=1, \operatorname{gcd}(i, n)=2$. Hence $i$ is an even and since $\operatorname{gcd}\left(i, \frac{n}{2}\right)=1$, it follows that $\frac{n}{2}$ is an odd which is denoted by $2 k+1$. Also $O\left(g^{i}\right)=n / 2=2 k+1$. Let $H$ be the subgroup of $G$ whose generated by $g^{i}$. We have $[G: H]=2$ and since $g^{n / 2} \notin H$, we have $G=H \cup H g^{n / 2}$. Observe that $H$ has an $n / 2$-cycle $e \sim a^{i} \sim a^{2 i} \sim \ldots \sim a^{(n / 2-1) i} \sim a^{n / 2 i}=e$ as a subgraph, in $\operatorname{Cay}(G, S)$. On the other hand, by Lemma [2.], $\mathrm{Hg}^{n / 2}$ has an $n / 2$-cycle as a subgraph, in $\operatorname{Cay}(G, S)$.

In the sequel, for each $1 \leq \ell \leq n / 2$, define $T_{\ell}=\left\{g^{\ell i}, g^{(\ell+n / 2) i}\right\}$ such that $g^{\ell i} \in H$ and $g^{(\ell+n / 2) i} \in$ $H g^{n / 2}$. Obviously, for each1 $\leq s<t \leq n / 2, T_{s} \cap T_{t}=\emptyset$. Since $g^{(\ell+n / 2) i} g^{-\ell i}=g^{n / 2} \in S$, induced subgraph on $T_{\ell}$ is isomorphic to $P_{2}$. In this case, $\operatorname{Cay}(G, S)$ is isomorphic to Figure 田.

In this case, since $n \geq 6$, Lemma [2.3 implies that $Z(\operatorname{Cay}(G, S)) \geq 4$. On the other hand, if $Z=\left\{e, g^{i}, g^{-i}, g^{n / 2}\right\}$, then it is easy to check that $Z$ is a zero forcing set for $\left.\operatorname{Cay}(G, S)\right)$ and so $Z(\operatorname{Cay}(G, S)) \leq 4$. Therefore, $Z(\operatorname{Cay}(G, S))=4$.

Question. Let $G$ be a group and $S$ be a subset of $G$ such that $S=S^{-1}$ and $e \notin S$. If induced subgraph on $S$ is empty, then is it true to say $Z(\operatorname{Cay}(G, S)) \geq 2|S|-2$ ? 


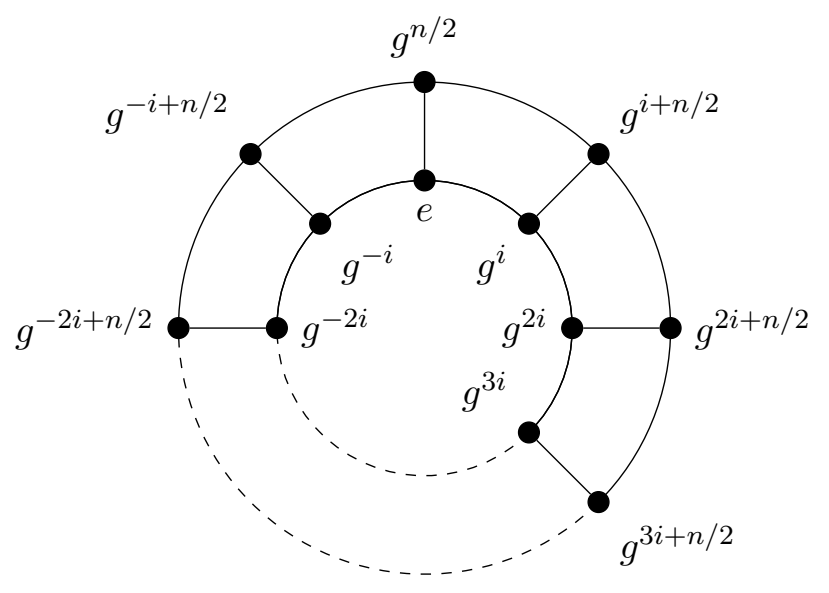

Figure 4. The Cartesian product $P_{2} \times C_{n}$.

\section{AcKnowledgments}

The authors are very grateful to the referee for his/her useful comments. This research was partially supported by the Imam Khomeini International University.

\section{REFERENCES}

[1] A. Abdollahi, E. Vatandoost, Which Cayley graphs are integral?, Electron. J. Combin., 16 (1) (2009), pp. $1-17$.

[2] AIM minimum rank-special graphs work group (F. Barioli, W. Barrett, S. Butler, S.M. Cioabă, D. Cvetković, S.M. Fallat, C. Godsil, W. Haemers, L. Hogben, R. Mikkelson, S. Narayan, O. Pryporova, I. Sciriha, W. So, D. Stevanović, H. van der Holst, K. Vander Meulen, A. Wangsness), Zero forcing sets and the minimum rank of graphs, Linear Algebra Appl., 428 (2008), pp. 1628-1648.

[3] F. Barioli, W. Barrett, S.M. Fallat, H.T. Hall, L. Hogben, B. Shader, P.V. Driessche, and H.V. Holst, Parameters related to tree-width, zero forcing, and maximum nullity of a graph, Journal of Graph Theory, 72(2) (2013), pp. 146-177.

[4] F. Barioli and S. Fallat, On the minimum rank of the join of graphs and decomposable graphs, Linear Algebra and its Applications, 421 (2007), pp. 252-263.

[5] F. Barioli, S. Fallat, and L. Hogben, A variant on the graph parameters of Colin de Verdière: Implications to the minimum rank of graphs, Electronic Journal of Linear Algebra, 13 (2005), pp. 387-404.

[6] A. Berman, S. Friedland, L. Hogben, U.G. Rothblum, B. Shader, An upper bound for the minimum rank of a graph, Linear Algebra and its Application, 429(2008), 1629-1638.

[7] R. Davila, F. Kenter, Bounds for the Zero-Forcing Number of Graphs with Large Girth, Theory and Applications of Graphs, 2(2) (2015), Article 1, pp. 1-8. 
Alg. Struc. Appl. Vol. 4 No. 2 (2017) 15-25.

[8] C.J. Edholm, L. Hogben, M. Huynh, J. LaGrange, D.D. Row, Vertex and edge spread of the zero forcing number, maximum nullity, and minimum rank of a graph, Linear Algebra and its Applications 436 (2012), pp. $4352-4372$.

[9] S.M. Fallat and L. Hogben, The minimum rank of symmetric matrices described by a graph: A survey, Linear Algebra and its Applications, 426 (2007), pp. 558-582.

[10] M. Gentner, L.D. Penso, D. Rautenbach, U.S. Souza, Extremal Values and Bounds for the Zero Forcing Number, Discrete Applied Mathematics, 214 (2016), pp. 196-200.

[11] M. Gentner and D. Rautenbach, Some Bounds on the Zero Forcing Number of a Graph, arXiv:1608.00747v1.

[12] L. Hogben, Orthogonal representations, minimum rank, and graph complements, Linear Algebra and its Applications, 428 (2008), pp. 2560-2568.

[13] C.R. Johnson, R. Loewy, and P.A. Smith, The graphs for which the maximum multiplicity of an eigenvalue is two, Linear and Multilinear Algebra, 57 (2007), pp. 713-736.

[14] D.D. Row, A technique for computing the zero forcing number of a graph with a cut-vertex, Linear Algebra and its Applications, 436 (2012), pp. 4423-4432.

\section{Ebrahim Vatandoost}

Department of Mathematics, Faculty of Science

Imam Khomeini International University

Qazvin, Iran.

vatandoost@sci.ikiu.ac.ir

\section{Yasser Golkhandy Pour}

Department of Mathematics, Faculty of Science

Imam Khomeini International University

Qazvin, Iran.

y.golkhandypour@edu.ikiu.ac.ir 